\title{
Advancing Trauma and Emergency Education Through Virtual Presence
}

\author{
Zachary M. Bauman ${ }^{1} \cdot$ Asad Azim $^{1} \cdot$ Rifat Latifi $^{1} \cdot$ Bellal Joseph $^{1}$
}

Published online: 28 June 2016

(C) Springer International Publishing AG 2016

\begin{abstract}
Purpose of Review Telemedicine has played a pivotal role in centralization of trauma care at large trauma centers and trauma systems. The purpose of this review is to highlight the role of telemedicine and virtual education in advancing trauma and emergency education through virtual presence

Recent Findings The use of telemedicine has recently revolutionized trauma patient care and education with the introduction of telepresence. The development of telepresence allows experienced trauma surgeons to have a virtual presence in emergency rooms in some of the most remote locations on the planet, providing up-to-date clinical knowledge and training to surgeons working in a remote area that is otherwise not possible.

Summary Through this virtual presence, trauma surgeons are able to help direct trauma patient care, advise on diagnostic studies and procedures, and help make appropriate decisions regarding the transfer of trauma patients.
\end{abstract}

Keywords Telemedicine $\cdot$ Telepresence $\cdot$ Teletrauma . Trauma care $\cdot$ Outcomes

\section{Introduction}

Injuries are a leading public health problem worldwide, accounting for an estimated $10 \%$ of global mortality $[1 \bullet \bullet, 2]$.

This article is part of the Topical Collection on Telemedicine and Innovative Remote Technologies for Trauma

Bellal Joseph

bjoseph@surgery.arizona.edu

1 Division of Trauma, Critical Care, Emergency Surgery, and Burns, Department of Surgery, University of Arizona, 1501 N. Campbell Ave, Room 5411, P.O. Box 245063, Tucson, AZ 85724, USA
Although trauma systems and major trauma centers have been shown to reduce morbidity and mortality $[3 \cdot \bullet, 4]$, most trauma specialists and trauma centers around the world are concentrated in urban settings $[3 \bullet \bullet, 5]$. In resource-poor and remote regions of the world, the lack of equipment, insufficient personnel, and minimal technical resources contribute to a less than optimal environment for acutely injured patients $[1 \bullet \bullet, 6]$. Although only $23-25 \%$ of the US population lives in rural America, $56.9 \%$ of deaths caused by motor vehicle crashes occur in this population [3••, 7]. Furthermore, it is estimated more than 600 people die or sustain long-term disability from traumatic injury in the USA each day with up to $40 \%$ of these deaths predicted as preventable if access to a well-organized trauma system was available uniformly $[8,9]$. Although it is difficult to determine one specific reason for such a large discrepancy in trauma care, there are a few factors that definitely contribute to this disparity. First, emergency room personnel in low volume trauma hospitals often have very limited experience with major traumatic injuries, potentially leading to management errors and deviation from standard of care practices. This is largely in part to the lack of training and continuous medical education in these canters $[8,10]$. Furthermore, in these remote environments, there is a lack of access to immediate subspecialty care such as trauma surgeons, neurosurgeons, orthopedic, vascular or cardiac surgeons hence delaying definitive care and limiting the ability for continuing education $[8,11]$.

Throughout time, man has been driven to develop new technology to make all aspects of people's lives more efficient, productive, and safe. This has definitely been the case in the field of medicine where our knowledge of the human body and how we can help when it is injured continues to expand exponentially. As our improved ability to care for the traumatically injured patient advances, so too must our ability to provide progressive care to all parts of the world. 
Amazing advances in communication technology, through the introduction of telepresence, have enhanced the delivery of trauma care around the world. Telemedicine can broadly be defined as the use of telecommunications and information technology to support the transfer and delivery of electronic medical data (i.e., high resolution images, radiographic images, sounds, live video, and patient records) from one location to another to provide healthcare at a distance [3••, 12•, 13•, 14]. The term "telepresence" describes the ability of health care providers to interact in real time with telecommunication equipment, allowing for immediate feedback and assistance. Telepresence, more specifically, is a subset of telemedicine in which a trauma surgeon is virtually present from a distant location during a real-time live experience [3••].

Trauma physicians and staff are now able to have a virtual presence in emergency and operating rooms around the world, guiding, educating, and collaboratively providing care to injured patients. This technology makes it possible for experienced trauma surgeons or specialists to assist or direct other less-experienced physicians who are operating on or caring for injured patients at a distance [3••]. Use of this new technology may provide access to medical care that might not otherwise be available to remote areas of the world as well as provide needed education for healthcare professionals [15]. Throughout this paper, we will discuss the important advancements that have been made in the care of the injured patient utilizing telemedicine and telepresence, and the implications for the future of trauma management and education.

\section{Smartphones in Trauma}

Smartphones have become extremely popular over the past few years, especially in the field of medicine. We know there are over 3 billion cellphone users in the world currently and this number continues to grow. The off-the-shelf technology of cellular phones has integrated its use in our daily lives. Many professions have integrated the use of smartphones to enhance and advance, and this has been seen greatest in medicine. Social media as well as multiple "mobile apps" have been an integral part of this advancement. The small size, simplicity of use, wide availability, obvious mobility, continuous Internet connectivity, and ease of telecommunication are valuable assets for the applicability of smartphones in telemedicine and virtual presence [16]. In addition, advancements in the ability of smartphones to take pictures or videos have played an integral role in management of the injured patient and trauma education [16]. In many institutions, photographs of patient injuries taken by smartphones have been used daily at sign-out sessions, transferred into patient medical records and utilized for follow-up appointments and continuity of care [16-18]. This application has enhanced the educational experience of all trauma staff and personnel allowing individuals to see various injuries and how they were managed as opposed to trying to verbally describe the situation. Furthermore, we have been able to use these images during multidisciplinary conferences to help consulting services understand the overall condition of the injured patient prior to, or after, their involvement in order to improve upon patient care. Finally, this application of smartphones allows for better sign-out between trauma teams giving a trauma team that did not see the patient initially the opportunity to become more familiar with the patient, leading to better care and enhanced educational opportunities [16-18]. Sign-outs are routinely a precarious time for patient care and resident education. A great deal of information can be lost during the sign-out process which eventually hurts resident education and training. The use of smartphones has not only made it easier to reach physicians and staff that have left the hospital, but photographs and videos have provided a new visual element to the communication, stressing the critical nature of a patient's condition.

Smartphones are also very suitable for assisting with teletrauma consultations. The camera provided on newer smartphones is far superior in quality to the old computerbased devices and appliances traditionally used in telemedicine [16]. The high-definition recording, ease of use, sophisticated features, and compact size of smartphones allows them significant mobility making them an extremely valuable tool for providing the best patient care through telemedicine, especially for healthcare providers on the go. A recent study by Joseph et al. demonstrated the number of teletrauma consultations increased substantially when rural hospital physicians and the on-call trauma surgeon at the urban-based Level I trauma center carried password-protected (in accordance with HIPPA regulations) smartphones as compared to older, stationary telemedicine systems [19••]. This study confirmed smartphones are a great method for trauma surgeons to continue their virtual presence in remote hospital locations to help provide better traumatic patient care. Not to mention, smartphones are cost-effective, easily replaceable and provide great mobility then other forms of telemedicine technology $[19 \bullet \bullet, 20]$. It is important to continue to integrate smartphones into the education and care of traumatic patients. As smartphones continue to evolve, the development of new "mobile apps" as well as continued advancements in smartphone virtual presence will not only improve trauma care, but all of healthcare throughout the world.

\section{Telepresence in Trauma}

Trauma telepresence, a concept evolved from telemedince, is when a trauma surgeon can have a virtual presence in trauma bays in remote locations assisting in the decision-making and care of an injured patient as well as providing additional trauma management education. Although advancements in 
technology now afford the ability to create an environment that mimics flawless motion, audio and video transmission, telepresence still remains underutilized as trauma systems worldwide are constantly figuring out ways they can incorporate this new technology [3••]. Trauma surgeon telepresence is now being used to identify knowledge gaps and the needs for instituting new outreach educational programs. As equipment is becoming more "user friendly" and less expensive, the perceptions of teletrauma and telepresence are evolving to become a vital component of modern care for the injured and acutely ill. It has successfully been used from simple surgical consultations with low bandwidth, to intraoperative guidance, to transatlantic spectacular surgical interventions, to disaster response scenarios. However, as more studies develop showing the benefits of telemedicine and telepresence, its utilization can only increase.

\section{Benefits of Telemedicine and Telepresence in Trauma}

Having the virtual presence of a trauma specialist in remote location emergency rooms has many advantages, not only for the patient but also for the team directly caring for the patient and the hospital system. These benefits are even seen if the patient is eventually transferred to the tertiary trauma center as the receiving physician would have been involved early on in the decision-making of the patient's care. Remote and underserved emergency departments usually do not see high volumes of trauma patients. Therefore, when a serious trauma presents itself, chaos and anxiety can ensue from inexperience. Having an experienced trauma surgeon virtually present in the emergency room that can see the patient and everything going on can help calm the team and make sure that management errors or deviation from standard of care does not occur [8]. Physicians and nurses have an ethical obligation to all patients to avoid causing further harm and provide optimal evidence-based care which may not always occur if the remote hospital does not see trauma patients on a regular basis [15]. During the initial assessment phase of a trauma, the virtual trauma surgeon may be able to defer time-consuming procedures and expedite transfer to the trauma center $[15,21]$. Furthermore, the consultant trauma surgeon can answer remote staff questions directly providing immediate feedback such as guidance on airway management, fluid resuscitation, early administration of blood products, and recommendation of best practices for reversal of therapeutic anticoagulation $[15,21,22]$. The trauma surgeon will also be able to visualize the patient and their injuries adding a fourth dimension to the decision-making process for patient care. Ultimately, telepresence facilitates a rapid and safe transfer from the remote emergency room to the urban trauma center avoiding untimely delays [15].

This leads to the next benefit of telemedicine and telepresence in trauma, which is that of providing continual medical education [8]. Debriefing after all trauma situations, hosting extracurricular teletrauma conferences, and being able to provide specific patient care feedback via telepresence allow remote hospital physicians and staff to continue to expand their knowledge and keep up with new trauma standards. Effective communication is key to any teletrauma system and must be present in order to provide appropriate and safe care for the patient. The common focus for any trauma situation should be the patient, and to achieve this, a nonhierarchical and collaborative relationship must exist between the virtual trauma surgeon/specialist and the remote hospital staff $[15,23]$. By improving communication and developing strong relationships with remote hospitals, continuing trauma education will be optimized and patient care will not suffer as a result.

One of the most beneficial aspects of having a trauma surgeon virtually present at remote hospitals is the decrease in costs to all entities involved, including the patient $[8,12 \cdot, 15]$. The experience at many trauma centers is that a large number of patients are transferred from remote hospitals to the trauma center when in fact they could be adequately managed at the remote hospital with the virtual presence of a trauma surgeon or specialist [8]. The cost of unnecessary transfers is significant not only to the patient but also to the accepting facility, as they must invest time and resources into caring for the patient when the patient could have been properly managed at the remote hospital. Furthermore, unnecessary transfers utilize emergency medical system providers that may otherwise be needed in the community tending to other patients. This is particularly seen in patients with soft tissue or burn wounds. A recent study by Latifi et al. demonstrated that with the implementation of a telemedicine and telepresence trauma system, 17 unnecessary transfers were prevented with an estimated $\$ 104,852$ in savings just from transfer costs alone (saving an average of $\$ 19,698$ per air transport and $\$ 2055$ per ground transport) [12•]. The savings from prevention of one unnecessary air transfer was enough to pay for the entire cost of one telemedicine system at the referring hospital (not including fixed costs such as Internet access, installation, and maintenance) [12•]. This is a very important point, as one of the biggest pushbacks to implementation of a telemedicine/ telepresence system is the start-up cost for the system itself. As demonstrated by this study, the cost savings accrued through implementation is significant and should not deter hospitals from developing a telemedicine/telepresence program.

Another study conducted by Duchesne et al. examined 814 injured patients seen at local community hospitals over a 5year period. The patients were divided into two groups: 315 patients before implementation and 463 patients after implementation of a telemedicine program with the university trauma center. Before implementation, all 351 injured patients were transferred to the university trauma center for definitive 
care. After implementation, however, all 463 patients received virtual consults resulting in only 51 patients being transferred to the university trauma center. Not only was this a huge cost savings to the health system of $\$ 6,505,941$, there was also a decreased length of stay for patients at the community hospitals by $45.5 \mathrm{~h}$ and a decrease in time to transfer to the university trauma center by $11.3 \mathrm{~h}[13 \bullet]$. Furthermore, the university trauma center was able to use their telemedicine program to facilitate the training and placement of specialty trained nurse practitioners in the rural community hospitals to work under direct clinical supervision by the emergency staff at the university trauma center via the telemedicine video link [13•]. This virtual presence is a great example of how teletrauma can not only make patient care more effective and efficient, but can reduce healthcare spending and provide education to allow for specialized trauma healthcare providers in remote hospitals.

Rural Vermont has also established a teletrauma system and has had great success thus far, especially in the area of decision-making for early disposition. In an early study of the teletrauma program in Vermont, 41 teletrauma consultations were completed, of which 31 patients were recommended for transfer to the trauma center and $59 \%$ of these transfers were recommended for immediate transfer given their critical nature $[10,24 \bullet]$. Furthermore, $15 \%$ of the consultations resulted in the trauma surgeon recommending keeping the patient at the rural hospital $[10,24 \bullet]$, a definite cost saving to the health system. Although patient disposition was the most common focus of these teletrauma consultations, other recommendations included whether to obtain additional imaging, placement of a chest tube or nasogastric tube, and administration of blood products [10, 24•]. Lambrecht also demonstrated the effectiveness of telemedicine in trauma through the evaluation of extremity and pelvic fractures $[3 \bullet \bullet, 25 \bullet \bullet]$. Orthopedic specialists evaluated 100 injured patients through telemedicine technology with an average of 8.6 min per teleconsult. Of these 100 patients, 68 were deemed appropriate to remain at the rural community hospital for care with zero significant adverse outcomes observed [25••]. Both of these studies further support the benefits of telemedicine/telepresence from not only a patient management and educational standpoint but also from an economical standpoint. Teletrauma has the potential for a large decrease in healthcare cost because of the lack of insurance in this patient population $[13 \cdot 26]$. Furthermore, treating patients away from their families and support system is definitely a tough situation for everyone.

Teletrauma extends well beyond the USA to other remote areas of the world. Over a 1-year period at the Aberdeen Royal Infirmary in Scotland, teleconsultations were performed for 120 patients: teleradiology was used for 116 patients, videoconferencing for 76 patients, and telepresence for 4 patients [27]. Use of their teletrauma system prevented 70 unnecessary transfers resulting in dramatic healthcare savings [27]. In
Taiwan, the use of video-telemedicine significantly reduced the frequency of unnecessary air medical transports from 16.1 to $0.1 \%$ with a total cost savings of US\$448,986 [1••, 28]. In Australia, a teleophthalmology program was successfully initiated where a digital slit lamp and fundus camera were utilized through the Internet for the diagnosis and management of traumatic and emergency ophthalmologic conditions $[1 \bullet \bullet, 29]$. A case series in Israel demonstrated video-conferencing was extremely useful to provide specialist care for acute trauma rapidly in a region of the world where armed conflict compromises accessibility and prevents direct physical access to care $[1 \bullet \bullet$, 30]. Furthermore, a case series in Canada demonstrated use of the Internet to successfully direct and observe the extended focused assessment with sonography for trauma (EFAST) $[1 \bullet \bullet, 31]$. Twenty total patients were analyzed with successful recognition of hemoperitoneum in five cases and pneumothoraces in two patients, not to mention the great educational benefits that accompanied the use of this teletrauma technology [31]. Finally, military medicine has been very interested in the potential of teletrauma given the obvious logistical problems involved during wartime. Telemedicine has already been successfully utilized in the Gulf War, Bosnia, and Macedonia [32-35] and as this technology continues to expand, so too will the military applications.

\section{Telemedicine/Virtual Presence in Intensive Care Medicine}

Tele-ICU is becoming more and more prevalent. The term teleICU is now used to describe an idea of healthcare in which a centralized critical care team is networked with the bedside intensive care unit (ICU) team and patient via audiovisual communication and computer systems. A recent review reported that formal ICU telemedicine programs now supervise about $11 \%$ of non-federal hospital critically ill adult patients and have demonstrated a decrease in hospital mortality and shorter ICU and hospital length of stay [36]. ICU telemedicine consists of remote surveillance of ICU patients using fixed installations, either continuously or during nighttime hours. Telemedicine can potentially improve ICU outcomes by increasing access to the experienced and board-certified intensivists and trauma surgeons facilitating early recognition of physiological deterioration, decision-making, and continuous education and training of healthcare providers working in remote or underdeveloped hospitals $[37,38]$. There are some logistic barriers to the broad adoption of ICU telemedicine, however, including the high technological costs as well as lack of consensus about how and where it is best applied.

A recent literature review of 91 studies on tele-ICU found that 46 of these studies assessed clinical outcomes, 36 assessed workload and staff acceptance, and 9 assessed cost and financial indicators. Of the 46 studies assessing clinical outcomes, a decrease in hospital length of stay and decrease in mortality were noted in all but 6 of the studies. Additional 
literature review of 25 tele-ICUs in the USA found that implementation of telemedicine programs favorably affected morbidity rates and length of stay [30,39, 40]. Future research and technological transformation that reduce costs and improve overall outcomes may change the implementation patterns of ICU telemedicine facilitating its adoption in more hospitals throughout the world.

\section{Telemedicine Functions for Trauma and Disaster Management}

The most common form of telemedicine/telepresence is that of inter-hospital programs, which we have spent a great deal of time discussing above. Another advanced technological function, however, is that of pre-hospital to hospital connectivity. This has been developed in the form of digital ambulances and monitored patient transport [3••]. At the Level 1 trauma center in Tucson, Arizona, collaboration with the Tucson Fire Department and Tucson Transportation Department has allowed for the development of a Wireless Mobile Telemedicine and Telepresence program in the pre-hospital setting by having video, audio, and data access available from various ambulances throughout the city $[3 \cdot \bullet]$. The telepresence at the scene of the event is provided via externally mounted cameras to the emergency vehicles [3••]. Furthermore, technology within the ambulances allows for constant two-way audio and visual transmission between ambulance personnel and the trauma and emergency room personnel $[3 \bullet \bullet]$. With this technology, the trauma and emergency staff can provide additional feedback to paramedics to help with stabilization of patients as well as give trauma and emergency room personnel a better idea of the mechanism of injury and ways that they can better prepare and care for the traumatically injured upon arrival. Furthermore, this technology has helped by assisting to clear accident scenes sooner, reducing the potential for secondary crashes $[3 \bullet \cdot]$.

Additionally, telemedicine and telepresence technology has advanced to the point where we can now provide deployable mobile telemedicine systems [3・•]. These deployable systems are compact enough to easily be carried by a few individuals and utilize email, telephone, and Internet services to provide telemedicine/telepresence with specialists all around the globe. Obvious application of this technology is for the military, briefly discussed above, however, other applications include expeditions to remote areas of the world (i.e., Mt. Everest) to help monitor individuals ensuring their safety and preventing additional injury or illness.

\section{Virtual Presence in Follow-up and Burn/Wound Care in Trauma Patients}

Another great application of telemedicine/telepresence is follow-up care for trauma patients. A study out of Kentucky assessed 22 trauma patients for follow-up utilizing a single nurse, electronic stethoscope, and close-up imaging instrumentation [41]. Average duration of each video-conference appointment was 14 min with both patient and physician satisfaction scores resulting very high [41]. Furthermore, these follow-up appointments demonstrated significantly decreased travel distances and time [41]. A survey was completed after these follow-up teleconference appointments and all patients involved agreed with the statement "Telemedicine makes it easier to get medical care" [41].

Telemedicine/telepresence has also shown promise in the field of wound care and care for the burn patient. A recent study demonstrated agreement between onsite and remote surgeons match for wound description and wound management. Sensitivity of remote diagnosis ranged from $78 \%$ for gangrene to $98 \%$ for identification of a problem with wound healing, respectively, whereas specificity ranged from $27 \%$ for erythema to $100 \%$ for recognition of ischemia [42]. Furthermore, in a recent review by Atiyeh et al. looking at the use of telemedicine and burn care, the feasibility of burn care to remote hospitals has been demonstrated throughout the world [43]. Not only has telemedicine/telepresence allowed the delivery of acute care to burn victims by guiding lessexperienced surgeons managing the patients, it has been employed for the long-term management of these patients, decreasing resource utilization and improving patient satisfaction [43]. Most importantly and similar to the acutely injured patient, it has allowed for better triage of burn patients, reducing unnecessary time and resource-demanding referrals that might overwhelm healthcare system capacities [43].

\section{Clinical Accuracy and Satisfaction of Telemedicine/Telepresence in Trauma}

The clinical accuracy of teletrauma has been assessed in a variety of studies. When telemedicine/telepresence was used for minor trauma consultation compared to face-to-face consultation in 200 patients, skin color changes were accurately defined in $97 \%$, the presence of swelling and deformity in $98 \%$, diminished joint movement in $95 \%$, presence of tenderness in $97 \%$, weight bearing and gait in $99 \%$, and radiologic diagnosis was made correctly in $98 \%$ of cases $[8,25 \bullet \cdot]$. Severity of injury was only overestimated in one case and underestimated in five cases $[8,25 \bullet \cdot]$. Other studies have similarly demonstrated that remote evaluation of trauma patients using telemedicine/telepresence was accurate and feasible [42, 44]. However, caution must be exercised when using teleradiology for subtle orthopedic injuries. Two recent studies did demonstrate differences in accuracy, sensitivity, and specificity with identification of subtle fractures utilizing teleradiology [45, 46]. Although it important to recognize these subtle fractures and address them appropriately, the likelihood of these subtle fractures being the difference between life and death is extremely low. As technology continues to 
improve, however, we anticipate this discrepancy will likely decrease.

One of the major issues that has been examined and continues to be a concern for those involved in telemedicine/ telepresence is that of patient and healthcare provider satisfaction. There have been a variety of studies conducted to analyze patient satisfaction with an overwhelming approval of the process, mainly because patients received high quality care without having to travel long distances away from their families to receive the care. When a study was conducted including 52 brain-injured patients that were interviewed via high quality teleconferencing, all the patients were very satisfied with their sessions and wanted to repeat it more than those patients interviewed in person [8, 25*0]. Furthermore, $83 \%$ of physicians at the remote hospitals and $61 \%$ of consulting trauma surgeons felt that the teletrauma consultations improved patient care with $67 \%$ of all physicians involved believing the consultation could not have been performed as well by telephone $[10,24 \bullet]$. This study further reinforces the effective and educational use of telemedicine/telepresence where both patients and the referring doctors benefited greatly from the expert opinion at a distance.

One of the major concerns of telemedicine/telepresence is that of liability. If the consultant is based in another state or country, certification and licensure requirements may differ [15]. Although this is a concern and that it is crucial all hospitals involved identify and address these variations (likely during program development), there have been no reported incidences to date of telemedicine/telepresence malpractice [12•]. It is more likely that malpractice would ensue without the telepresence of a specialist, especially if standard of care is not followed at the remote hospital. Furthermore, some states are now paying for telemedicine consultations, relieving some of the liability of the remote hospital and staff [12•].

\section{Conclusion}

The evidence, advancement, and effectiveness of teletrauma and telepresence are unquestionable, despite the previous initial high cost of setting up these systems. These services will only continue to grow with the advancement of off-the-shelf technology that has revolutionized communication technology. Telemedicine and telepresence is and will continue to be a major tool not only in the initial care of traumatically injured patients but also for in-patient critical care, trauma education and training, and trauma patient follow-up. Current teletrauma technologically allows consulting trauma surgeons to provide guidance to remote hospitals in terms of diagnostic studies, procedures to be completed, blood product administration, and overall disposition for the injured patient. Studies have repetitively demonstrated that teletrauma is now more costeffective than before by preventing unnecessary transfers.
Furthermore, teletrauma is potentially life-saving by promoting early transfer when medically necessary. Teletrauma has also shown to be safe for both remote physicians providing care as well as the patients receiving care and it has improved patient satisfaction by decreasing time patients are away from family. As telemedicine/telepresence continues to develop, so too will its application in trauma care across multiple disciplines.

Telehealth technology has the latent potential of refining healthcare training, education, and research. Key improvements will emerge from research and further development of telemedicine, and with incorporation of these technologies into the healthcare system, standards of healthcare can be revolutionized and homogenized. Federal health institutes can further enhance utility and effectiveness of telemedicine by playing a lead role in facilitating telehealth innovation, establishing specific research goals, setting up collaboration/ demonstration centers, and facilitating access to cyber-infrastructure. These efforts will not only enhance healthcare training, education, and research activities across the USA but around the world.

\section{Compliance with Ethical Standards}

Conflict of Interest Drs. Bauman, Azim, Latifi, and Joseph declare no conflicts of interest relevant to this manuscript.

Human and Animal Rights and Informed Consent This article does not contain any studies with human or animal subjects performed by any of the authors.

Funding There are no financial interests or funding to disclose for any of the contributing authors.

\section{References}

Papers of particular interest, published recently, have been highlighted as:

- Of importance

•. Of major importance

1.• Lewis ER, Thomas CA, Wilson ML, Mbarika VW. Telemedicine in acute-phase injury management: a review of practice and advancements. Telemed J E Health. 2012;18(6):434-45. This manuscript is of great importance because it highlights different ways in which telemedicine is creatively being implemented worldwide to help provide better patient care and improve healthcare cost savings.

2. Bhalla K, Harrison JE, Shahraz S, Fingerhut LA. Availability and quality of cause-of-death data for estimating the global burden of injuries. Bull World Health Organ. 2010;88:831-838C.

3.• Latifi R, Weinstein RS, Porter JM, Ziemba M, Judkins D, Ridings $\mathrm{D}$, et al. Telemedicine and telepresence for trauma and emergency care management. Scand J Surg. 2007;96:281-9. This manuscript 
is of great importance because it lays a foundation for three of the major ways in which telemedicine/telepresence can be utilized to improve trauma care. Most telemedicine/telepresence programs that have been developed have utilized some form of the programs discussed in this paper.

4. Trunkey DD. Trauma centers and trauma systems. JAMA. 2003;289(12):1566-7.

5. Branas CC, MacKenzie EJ, Williams JC, Schwab CW, Teter HM, Flanigan MC, et al. Access to trauma centers in the United States. JAMA. 2005;293(21):2626-33.

6. Peden M, Oyegbite K, Ozanne-Smith J, Hyder AA, Branche C, Rahman AKMF, et al. World report on child injury prevention. Geneva: World Health Organization; 2008.

7. Congressional Office of Technology Assessment: Rural Emergency Medical Services. Special Reports US, Washington, DC, 1989. (Publication OTA-H-445)

8. Latifi R, Peck K, Porter JM, Poropatich R, Geare T, Nassi RB. Telepresence and telemedicine in trauma and emergency care management. Stud Health Technol Infrom. 2004;104:193-9.

9. Voelker R. Access to trauma care. JAMA. 2000;284(16):2048.

10. Ricci MA, Caputo M, Amour J, Rodgers FB, Sartorelli K, Callas $\mathrm{PW}$, et al. Telemedicine reduces discrepancies in rural trauma care. Telemed J E Health. 2003;9:3-11.

11. Flow KM, Cunningham PRG, Foil MB. Rural trauma. Ann Surg. 1995;27:29.

12. Latifi R, Hadeed GJ, Rhee P, O'Keeffe T, Friese RS, Wynne JL, et al. Initial experiences and outcomes of telepresence in the management of trauma and emergency surgical patients. Am J Surg. 2009;198:905-10. These three manuscripts are of importance because these are the three implemented teletrauma programs that have been written about here in the United States. All three demonstrate how teletrauma has improved patient care, education and have been economically smart for their state's trauma system. Furthermore, these manuscripts also discuss how the teletrauma programs were implemented. Any trauma system looking to set up a teletrauma program should definitely read these papers.

13. Duchesne JC, Kyle A, Simmons J, Islam S, Schmieg RE, Olivier J, et al. Impact of telemedicine upon rural trauma care. J Trauma. 2008;64:92-8. These three manuscripts are of importance because these are the three implemented teletrauma programs that have been written about here in the United States. All three demonstrate how teletrauma has improved patient care, education and have been economically smart for their state's trauma system. Furthermore, these manuscripts also discuss how the teletrauma programs were implemented. Any trauma system looking to set up a teletrauma program should definitely read these papers.

14. Wootton R. Telemedicine. BMJ. 2001;323:557-60.

15. Wesson JB, Kupperschmidt B. Rural trauma telemedicine. J Trauma Nurs. 2013;20(4):199-202.

16. Zangbar B, Pandit V, Rhee P, Aziz H, Hashmi A, Friese RS, et al. Smartphone surgery: how technology can transform practice. Telemed J E Health. 2014;20(6):590-2.

17. Joseph B, Pandit V, Wynn J, Aziz H, Tang A, Kulvatunyou N, et al. Telephotography in trauma: a 2-year clinical experience. Telemed J E Health. 2014;20(4):342-5.

18. Joseph B, Pandit V, Khreiss M, Aziz H, Kulvatunyou N, Tang A, et al. Improving communication in level 1 trauma centers: replacing pagers with smartphones. Telemed J E Health. 2013;19(3):150-4.

19.•- Joseph B, Hadeed G, Sadoun M, Rhee PM, Weinstein RS. Video consultation for trauma and emergency surgical patients. Crit Care Nurs Quart. 2012;35(4):341-5. This manuscript is of great importance because it is one of the first published manuscripts demonstrating the use of smartphones to improve communication between rural hospitals and a level 1 trauma center. The use of the video function and photography allowed for better patient care and disposition, as well as improved physician satisfaction. Furthermore, this study demonstrated that because of the small size of smartphones, they are ideal for telemedicine because of their mobility, ease of replacement and the fact that they are not very expensive.

20. Katzir M, Laarakker AS, Sviri GE, Zaaroor M. Smart(phone) Medicine. J Trauma Acute Care Surg. 2015;80(1):171-2.

21. Bjorn P. Rural teletrauma applications, opportunities and challenges. Adv Emerg Nurs J. 2012;34(3):232-7.

22. Schulman CI, Marttos A, Rothenberg P, Augenstein J. Usability of telepresence in a level 1 trauma center. Telemed J E Health. 2013;19(4):248-51.

23. Crawford CL, Omery A, Seago JA. The challenges of nursephysician communication; a review of the evidence. J Nurs Adm. 2012;42(12):548-50.

24. Rogers F, Ricci M, Caputo M, Shackford S, Sartorelli K, Callas P, et al. The use of telemedicine for real-time video consultation between trauma center and community hospital in a rural setting improves early trauma care: preliminary results. J Trauma. 2001;51(6):103741. These three manuscripts are of importance because these are the three implemented teletrauma programs that have been written about here in the United States. All three demonstrate how teletrauma has improved patient care, education and have been economically smart for their state's trauma system. Furthermore, these manuscripts also discuss how the teletrauma programs were implemented. Any trauma system looking to set up a teletrauma program should definitely read these papers.

25.• Lambrecht CJ. Telemedicine in trauma care: description of 100 trauma teleconsults. Telemed J. 1997;3(4):265-8. This manuscript is of great importance because it was one of the early papers to demonstrate the clinical accuracy of telemedicine as well as increased patient satisfaction using telemedicine.

26. Launois R. Economic aspects of the telemedicine. Bull Acad Natl Med. 2006;190:367-77.

27. Armstrong IJ, Haston WS. Medical decision support for remote general practitioners using telemedicine. J Telemed Telecare. 1997;3:27-34.

28. Tsai SH, Kraus J, Wu HR, Chen WL, Chuang MF, Lu LH, et al. The effectiveness of video-telemedicine for screening of patients requesting emergency air medical transport (EAMT). J Trauma. 2007;62(2):504-11.

29. Kumar S, Yogesan K, Hudson B, Tay-Kearney ML, Constable IJ. Emergency eye care in rural Australia: role of Internet. Eye (Lond). 2006;20:1342-4.

30. Kumar S, Merchant S, Reynolds R. Tele-ICU: efficacy and costeffectiveness approach of remotely managing the critical care. Open Med Inform J. 2013;7:24-9.

31. Dyer D, Cusden J, Turner C, Boyd J, Hall R, Lautner D, et al. The clinical and technical evaluation of a remote telementored telesonography system during the acute resuscitation and transfer of the injured patient. J Trauma. 2008;65:1209-16.

32. Calcagni DS, Clyburn CA, Tomkins G, Gilbert GR, Cramer TJ, Lea RK, et al. Operation joint endeavor in Bosnia: telemedicine systems and case reports. Telemed J. 1996;2:211-24.

33. Vassallo DJ, Buxto PJ, Kilbey JH, Trasler M. The first telemedicine link for the British forces. J R Army Med Corps. 1998;144:125-30.

34. Person DA. Telemedicine in cyberspace. Mil Med Technol. 1998;2: 13-5.

35. LeVec LN. Medics in reserve. Mil Med Technol. 1998;2:32-5.

36. Society of Critical Care Medicine Tele-ICU Committee. Critical care telemedicine: evolution and state of the art. Crit Care Med. 2014;42(11):2429-36. 
37. Lilly CM, Fisher KA, Ries M, Pastores SM, Vender J, Pitts JA, et al. A national ICU telemedicine survey: validation and results. Chest. 2012;142:40-7.

38. Pronovost PJ, Angus DC, Dorman T, Robinson KA, Dremsizov TT, Young TL. Physician staffing patterns and clinical outcomes in critically ill patients: a systematic review. JAMA. 2002;288:2151-62.

39. Ramnath VR, Ho L, Maggio LA, Khazeni N. Centralized monitoring and virtual consultant models of tele-ICU care: a systematic review. Telemed J E Health. 2014;20:936-61.

40. Ramnath VR, Khazeni N. Centralized monitoring and virtual consultant models of tele-ICU care: a side-by-side review. Telemed J E Health. 2014;20(10):962-71.

41. Tachakra S, Jaye P, Bak J, Hayes J, Sivakumar A. Supervising trauma support by telemedicine. J Telemed Telecare. 2000;6 suppl $1: 7-11$.
42. Tachakra S, Lynch M, Newsom R, Stinson A, Sivakumar A, Hayes $\mathrm{J}$, et al. A comparison of telemedicine with face-to-face consultations for trauma management. J Telemed Telecare. 2000;5 suppl 1: S178-81.

43. Atiyeh B, Dibo SA, Janom HH. Telemedicine and burns: an overview. Ann Burns Fire Disasters. 2014;27(2):87-93.

44. Wirthlin DJ, Buradagunta S, Edwads RA, Brewster DC, Cambria RP, Gertler JP, et al. Telemedicine in vascular surgery: feasibility of digital imaging for remote management of wounds. J Vasc Surg. 1998;27(6):1089-99.

45. Wilson AJ, Hodge JC. Digitalized radiographs in skeletal trauma: a performance comparison between a digital workstation and the original film images. Radiology. 1995;196:565-8.

46. Scott WW, Rosenbaum JE, Ackerman SJ, Reichle RL, Magid D, Weller JC, et al. Subtle orthopedic fractures: teleradiology workstation versus film interpretation. Radiology. 1993;187:811-5. 